\title{
Limits on Source Distances for the Most Energetic Cosmic Rays
}

\author{
R. Lampard, R. W. Clay and B. R. Dawson \\ Department of Physics and Mathematical Physics, University of Adelaide, \\ Adelaide, SA 5005, Australia \\ rclay@physics.adelaide.edu.au \\ Received 1997 January 16, accepted 1997 July 12
}

\begin{abstract}
The propagation of the highest energy cosmic rays through the $2.7 \mathrm{~K}$ microwave background is considered. Photo-pion production interactions cause energy losses for primary cosmic ray protons which result in significant energy degradation over intergalactic distances. The process of energy loss is discussed and an estimate is made of the average distances travelled for observed cosmic rays with a range of energies, assuming a power law source spectrum. At energies over $10^{20} \mathrm{eV}$, the average distances travelled are a few tens of megaparsecs, limiting possible sources to the volume dominated by the supergalactic plane.
\end{abstract}

Keywords: cosmic rays: general - ISM: cosmic rays

\section{Introduction}

Cosmic rays have been observed with energies up to $3 \times 10^{20} \mathrm{eV}$ (Bird et al. 1995) yet, to the present time, there is no agreed source for these particles. Though not certain, there is evidence that the flux is dominated by protons (Bird et al. 1993) with fewer heavier nuclei than exist at lower cosmic ray energies. It has recently been noted (Stanev et al. 1995) that directional data from the northern hemisphere observatories suggest a correlation of their arrival directions with the direction of the supergalactic plane. On the other hand, data from a southern experiment (Kewley, Clay \& Dawson 1996) do not confirm this.

It is expected that the highest energy cosmic ray protons will interact with the $2.7 \mathrm{~K}$ microwave background mainly through photo-pion production. This will limit the possible distances to their sources and result in a corresponding distortion of the energy spectrum above a few times $10^{18} \mathrm{eV}$. There has been a general concentration of interest in the form of the spectrum but, since these particles are likely to have trajectories which are approaching straight lines through the intergalactic magnetic fields and, since there is also a proposed source region (the supergalactic plane), we wish to examine the limitations to the source distance imposed by interactions with the $2 \cdot 7 \mathrm{~K}$ cosmic microwave background. This topic has been examined most recently by Elbert \& Sommers (1995) and by Geddes, Quinn \& Wald (1996) and, where they are comparable, our results are in agreement. We emphasise here the component parts of such calculations and the astrophysical consequences of the results.
2 Interactions between the Highest Energy Cosmic Rays and the 2.7 K Background.

The energies of the cosmic microwave background (CMB) photons are distributed according to the Planck formula:

$$
n_{\gamma}(E) d E=\frac{8 \pi}{h^{3} c^{3}} \times \frac{E^{2} d E}{\exp (E / k T)-1} .
$$

Cosmic Background Explorer Satellite measurements give $T=2 \cdot 735 \pm 0.06 \mathrm{~K}$ (Mather et al. 1990). Integrating over all energies gives the total number density of 415 photons $/ \mathrm{cm}^{3}$ for $T=2.735 \mathrm{~K}$. The bulk of the microwave photons have energies below $2.5 \times 10^{-3} \mathrm{eV}$ with the mean energy being $6 \cdot 37 \times 10^{-4}$ $\mathrm{eV}$. In the cosmic ray rest frame, the low energy $2 \cdot 7 \mathrm{~K}$ photons appear as gamma-rays with energies $>100 \mathrm{MeV}$ and can result in energy degradation of the cosmic ray particle. Pion photo-production and pair production are the two most important processes for interactions between ultra high energy (UHE) cosmic ray protons and photons, in terms of the size of the cross sections as seen by the cosmic ray. The fraction of energy lost in a single interaction by the cosmic ray proton due to pion production ranges from $15 \%$ to $50 \%$. By contrast, the energy lost in a single interaction due to pair production $\left(p+\gamma \rightarrow p+e^{+}+e^{-}\right)$is of the order $m_{e} / m_{p} \sim 0 \cdot 1 \%$, where $m_{e}$ and $m_{p}$ are the electron and proton masses respectively. Therefore, energy losses due to pair production can be neglected.

The pion production processes for a proton are

$$
p+\gamma \rightarrow p+\operatorname{pion}(\mathrm{s})
$$

$1323-3580 / 97 / 030258 \$ 05.00$ 


$$
p+\gamma \rightarrow n+\operatorname{pion}(\mathrm{s})
$$

In roughly half of these interactions the proton is transformed into a neutron. The interaction of a neutron (in its own instantaneous rest frame) with a high energy photon will also result in pion production:

$$
\begin{aligned}
& n+\gamma \rightarrow n+\operatorname{pion}(\mathrm{s}) \\
& n+\gamma \rightarrow p+\operatorname{pion}(\mathrm{s})
\end{aligned}
$$

Unlike the $\gamma p$ cross section, the $\gamma n$ cross section cannot be experimentally determined because of the short mean lifetime ( $15 \mathrm{~min}$, Humble 1990) of free neutrons. Here, following Hill and Schramm (1985), it was inferred by taking the difference between the $\gamma d$ and $\gamma p$ cross sections (where $d$ is deuterium).

In the following sections, the calculation of the mean free path and inelasticity is discussed. Note, that while protons are referred to, the discussion also applies to neutrons.

\subsection{Calculation of the Mean Free Path}

The starting point for the calculation of the mean free path $\lambda$ is the relation:

$$
\lambda=\frac{1}{n \sigma},
$$

where $\sigma$ is the cross section for an interaction between some incident particle and a target particle, and there are $n$ target particles per unit volume. In this case, $n$ is simply the number density of $2 \cdot 7 \mathrm{~K}$ microwave photons.

To determine what value to use for $\sigma$, the relation between the photon energy in the lab frame (where an observer sees $\mathrm{a} \sim 10^{20} \mathrm{eV}$ proton collide with $\mathrm{a}$ microwave photon) and the photon energy in the proton rest frame (in which the individual cosmic ray is stationary) has to be considered. This is given by the Doppler formula:

$$
E^{\prime}=\gamma E(1-\beta \cos \theta)
$$

where $E^{\prime}$ is the photon energy in the proton rest frame and $E$ is the microwave (lab frame) photon energy. Here $\gamma=E_{p} / m_{p} c^{2}$ is the gamma factor, with $E_{p}$ and $m_{p}$ being the cosmic ray energy and proton rest mass, while $\theta$ is the angle between the momentum vectors of the cosmic ray and microwave photon in the lab frame. For UHE cosmic rays $\beta(=v / c)$ can be taken to be equal to 1 .

It follows from the Doppler relation that the cross section as seen by the cosmic ray will depend on both $E$ and $\theta$. Hence in a calculation of $\lambda\left(E_{p}\right)$, a mean value for the cross section has to be used.
This is found by averaging $\sigma$ over all possible values of $E$ and $\theta$ for each particular $E_{p}$.

In deriving an expression for $\langle\sigma\rangle$, the mean cross section, we account for this and find:

$$
\begin{aligned}
\langle\sigma\rangle= & \frac{4 \pi}{\gamma^{2} h^{3} c^{3} N_{\gamma}} \int_{0}^{\infty} d E \frac{1}{\exp (E / k T)-1} \\
& \times \int_{0}^{2 \gamma E} d E^{\prime} \cdot E^{\prime} \sigma\left(E^{\prime}\right)
\end{aligned}
$$

where $N_{\gamma}$ is the number density of microwave background photons.

In the proton rest frame there is a threshold energy $\left(E^{\prime}{ }_{t h r}=145 \mathrm{MeV}\right)$ of the gamma-ray photons below which pion photo-production does not occur. It follows that in the lab frame, microwave photons with energies below a certain value $E_{c}$ will not cause pion photo-production even for a head-on collision. The Doppler relation then gives

$$
E_{c}=\frac{145 \mathrm{MeV}}{2 \gamma} .
$$

Below the threshold, the cross section is negligible. Therefore in a numerical calculation of $\langle\sigma\rangle$ the values $E_{t h r}^{\prime}$ and $E_{c}$ can be chosen as lower limits for the integrations over $E^{\prime}$ and $E$ respectively. A conservative upper limit of $10^{-2} \mathrm{eV}$ was chosen for the integration over $E$ by noting that above an energy of about $2.5 \times 10^{-3} \mathrm{eV}$ the number of microwave photons is negligible.

An expression for the mean free path follows from equations (2) and (4):

$$
\begin{aligned}
\lambda= & \frac{\gamma^{2} h^{3} c^{3}}{4 \pi}\left[\int_{0}^{\infty} d E \frac{1}{\exp (E / k T)-1}\right. \\
& \left.\times \int_{0}^{2 \gamma E} d E^{\prime} \cdot E^{\prime} \sigma\left(E^{\prime}\right)\right]^{-1}
\end{aligned}
$$

\subsection{Calculation of the Inelasticity}

In our Monte Carlo calculations we sample the microwave background photon energy distribution and then determine its energy in the rest frame of the cosmic ray. There are two factors that will determine the probability for a particular angle of incidence. One is the directional dependence of the photon density as seen by the cosmic ray due to its motion through the $\mathrm{CMB}$, which will result in a higher probability for head-on collisions. The second factor is the size of the cross section as seen by the cosmic ray, which will be a function of the angle of incidence of the CMB photon. The cross section is dominated by a large resonance peak just above threshold, hence angles of incidence such that the cosmic ray sees this resonance peak will 


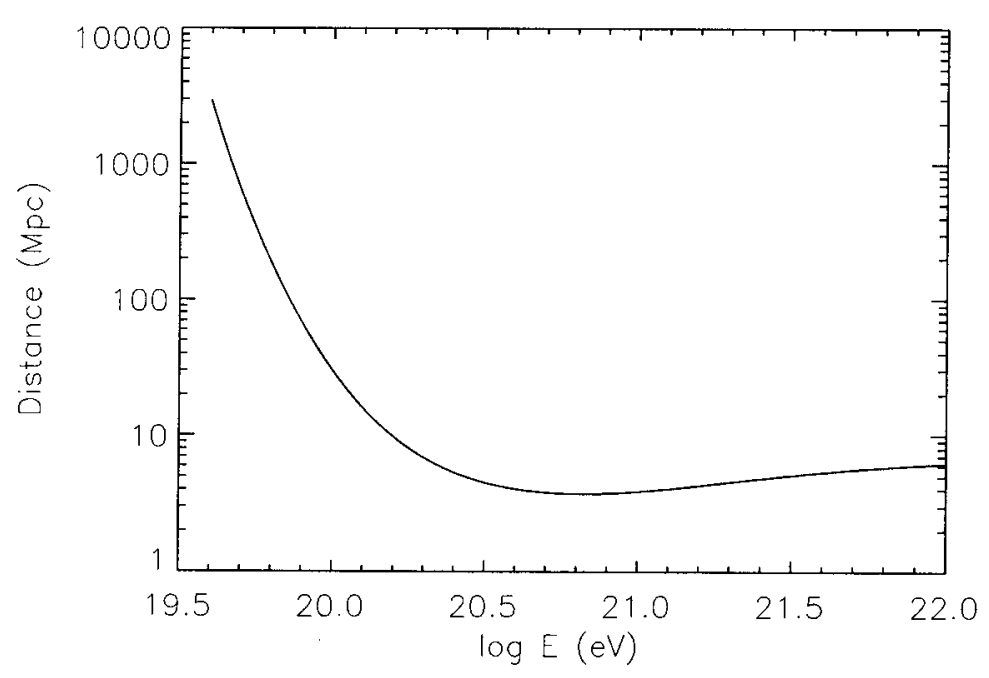

Figure 1-Mean free path for interaction of a proton with the CMB.

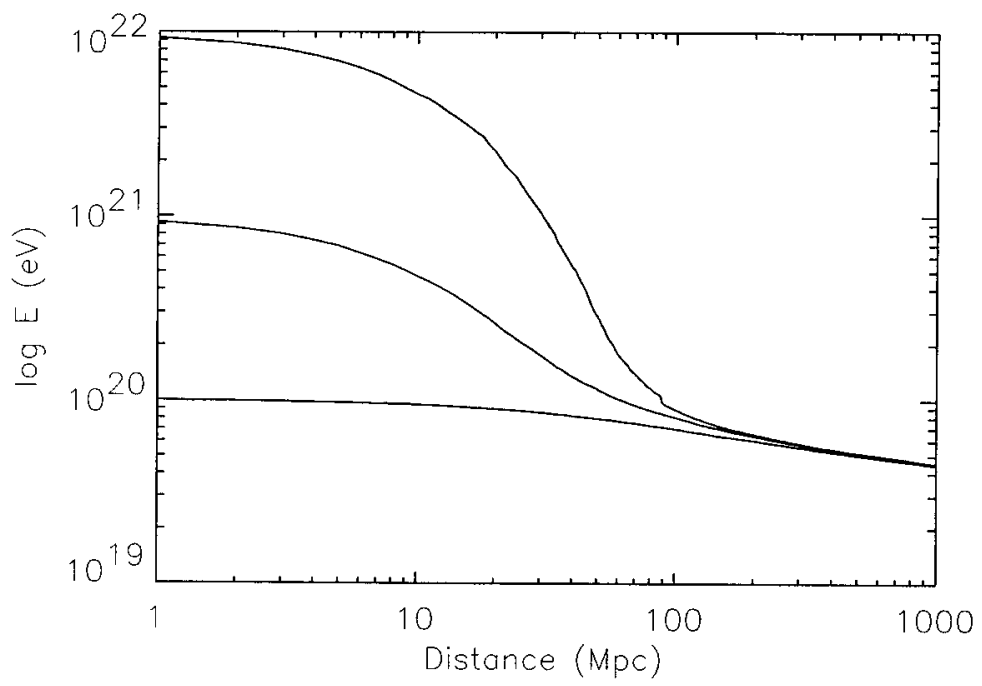

Figure 2-Mean energy as a function of distance, of a sample of 1000 protons, with initial energies as indicated.

be favoured. The combination of these two factors results in the probability distribution

$P\left(E^{\prime}\right)=E^{\prime} \sigma\left(E^{\prime}\right) \times\left[\int_{0}^{2 \gamma E} d E^{\prime} \cdot E^{\prime} \sigma\left(E^{\prime}\right)\right]^{-1}$.

In the Monte Carlo calculation, a value of $E^{\prime}$ between $E_{t h r}^{\prime}=145 \mathrm{MeV}$ and $E_{\text {max }}^{\prime}=2 \gamma E$ is then sampled from this distribution.

The inelasticity is the fraction of energy lost by a particle in an interaction. It is given by

$$
K=\frac{\Delta E}{E}
$$

where $E$ is the initial energy and $\Delta E$ is the energy loss. A Monte Carlo technique was used to compute the inelasticity for the case of single pion production. For a particular interaction, the CMB photon energy and angle of incidence (in the lab frame) were randomly chosen. On the creation of a pion, energy is lost by the cosmic ray. This energy loss was calculated in the centre of momentum (COM) frame.

The energy of the photon in the cosmic ray rest frame is given by the Doppler formula, while the angle of incidence can be determined from the aberration formula:

$$
\cos \phi=\frac{\cos \theta-\beta}{1-\beta \cos \theta}
$$

For cosmic rays with energies $\sim 10^{20} \mathrm{eV}, \beta$ is close to 1 and thus $\cos \phi$ is close to -1 . This means 
that in the cosmic ray rest frame the photon can be considered as travelling along the $x$-axis towards the cosmic ray. In other words, the cosmic ray will always see the photon coming in from a head-on direction, no matter what the angle of incidence in the lab frame. In the COM frame, the cosmic ray and photon are required to have oppositely directed momenta of equal magnitude. Once the proton energy and momentum in the COM frame are known, they can be transformed back to the lab frame, via the rest frame, to give the final energy of the cosmic ray. The above procedure can be used to relate the initial and final energies of a cosmic ray that has been involved in a pion photo-production interaction and thus find the mean inelasticity as a function of cosmic ray energy:

$$
\begin{aligned}
E_{f}=E_{0} & {\left[\frac{s+\left(m_{p}^{2}-m_{\pi}^{2}\right)}{2 s}\right.} \\
& \left.+\cos \theta \sqrt{\frac{\left[s+\left(m_{p}^{2}-m_{\pi}^{2}\right)\right]^{2}}{4 s^{2}}-\frac{m_{p}^{2}}{s}}\right]
\end{aligned}
$$

where $E_{0}$ is the initial cosmic ray energy, $E_{f}$ is the final cosmic ray energy, and $\theta$ is the emission angle of the nucleon in the COM frame. The Lorentz invariant $s$ can be calculated from $E^{\prime}$ :

$$
s=m_{p}^{2}+2 m_{p} E^{\prime}
$$

The above method to calculate the inelasticity deals with single pion production only. However, it can be extended to include multiple pion production, in which case it is necessary to use the actual experimental data, which gives the distribution of emission angles and energies of the pions. Thus, in a Monte Carlo calculation, values for the pion emission angles and energies are randomly sampled, from which the inelasticity of the interaction is calculated (see Szabo 1992; Protheroe \& Johnson 1996). In this paper, the approximation is made that an interaction of a cosmic ray with a CMB photon always results in single pion production. Aharonian \& Cronin (1994) found that the assumption that only single pion production occurs does not introduce significant error into the results when modelling the propagation of UHE cosmic rays through the CMB.

\subsection{The Mean Free Path}

In Figure 1 the mean free path for interaction of a proton with the microwave background (calculated from the expression derived earlier) is plotted as a function of cosmic ray energy. Clearly, cosmic rays with energies above $10^{20} \mathrm{eV}$ will be strongly attenuated by interactions with the CMB if they are propagated over distances of more than a few megaparsecs. Thus, it can be inferred that any protons detected with such an energy have a probable origin within a distance of a few tens of Mpc. Cosmic ray protons with energies in the range $6 \times 10^{19}$ to $10^{20} \mathrm{eV}$ are also attenuated by interactions with microwave photons, but not so strongly. It is in this energy range that the mean free path $\lambda$ increases dramatically as the energy drops, such that below an energy of about $5 \times 10^{19} \mathrm{eV}, \lambda$ is of the order of thousands of megaparsecs. The corresponding mean time between interactions is of the same order as the age of the universe.

\subsection{Energy Attenuation}

Figure 2 shows the attenuation in energy of cosmic ray protons due to interactions with the microwave photons. For each of the three initial energies shown, a sample of 1000 protons were propagated through the microwave background with a step size of one megaparsec. For each step, the probability per unit length $(1 \mathrm{Mpc})$ of interaction was computed from the mean free path and any resulting energy loss calculated using the Monte Carlo techniques discussed above. After each increment the proton energy was recorded. The figure shows the mean energy of a particular sample as a function of propagation distance. The result agrees with that given by Aharonian \& Cronin (1994).

\section{Source Distance Distributions}

We now wish to discuss the results of a Monte Carlo calculation of source distance distributions. In this calculation, a sample of UHE protons is propagated through the CMB. The initial energy of a particular proton is randomly chosen from a $E^{-2}$ differential spectrum, with an energy between $8 \times 10^{19}$ and $10^{22}$ $\mathrm{eV}$. The particle is propagated through the $\mathrm{CMB}$ until its energy is degraded to below $8 \times 10^{19} \mathrm{eV}$. At random points along its trajectory (which in the absence of a magnetic field is a straight line), the proton interacts with CMB photons and loses energy. At each of these points, the proton's energy (just before interaction) and the distance from the origin (i.e. source) is recorded.

The distance values are binned according to the degraded value of the energy. Thus, for a large sample of protons, a distribution will be obtained for each energy bin. These distributions are of the distances a cosmic ray proton selected from a power law source spectrum will propagate, in the absence of a magnetic field, such that its observed energy is in the range $E$ to $E+\Delta E$.

For each distance distribution the mean was calculated. Since the distributions are not symmetric about the mean, the standard deviations were not computed. Instead, the $15 \%$ and $85 \%$ points of the integral distribution were determined. 
Table 1. Distances from source at various energies for $10^{5}$ particles, with initial energies sampled from an $E^{-2}$ differential spectrum that truncates at $10^{22} \mathrm{eV}$

The $15 \%$ and $85 \%$ points, and the mean, are given to the nearest $\mathrm{Mpc}$

\begin{tabular}{|c|c|c|c|c|}
\hline $\operatorname{Bin}(e V)$ & Number of data values & Mean (Mpc) & $15 \%$ point $(\mathrm{Mpc})$ & $85 \%$ point $(\mathrm{Mpc})$ \\
\hline $8-9 \times 10^{19}$ & 49578 & 104 & 32 & 177 \\
\hline $9-10 \times 10^{19}$ & 39016 & 74 & 23 & 126 \\
\hline $1-2 \times 10^{20}$ & 162809 & 37 & 9 & 65 \\
\hline $2-3 \times 10^{20}$ & 47219 & 17 & 4 & 30 \\
\hline $3-4 \times 10^{20}$ & 21619 & 14 & 3 & 24 \\
\hline $4-5 \times 10^{20}$ & 11989 & 13 & 2 & 23 \\
\hline $5-6 \times 10^{20}$ & 7566 & 13 & 2 & 22 \\
\hline $6-7 \times 10^{20}$ & 5011 & 11 & 2 & 21 \\
\hline $7-8 \times 10^{20}$ & 3542 & 11 & 2 & 21 \\
\hline $8-9 \times 10^{20}$ & 2700 & 11 & 2 & 20 \\
\hline $9-10 \times 10^{20}$ & 2137 & 11 & 2 & 20 \\
\hline
\end{tabular}
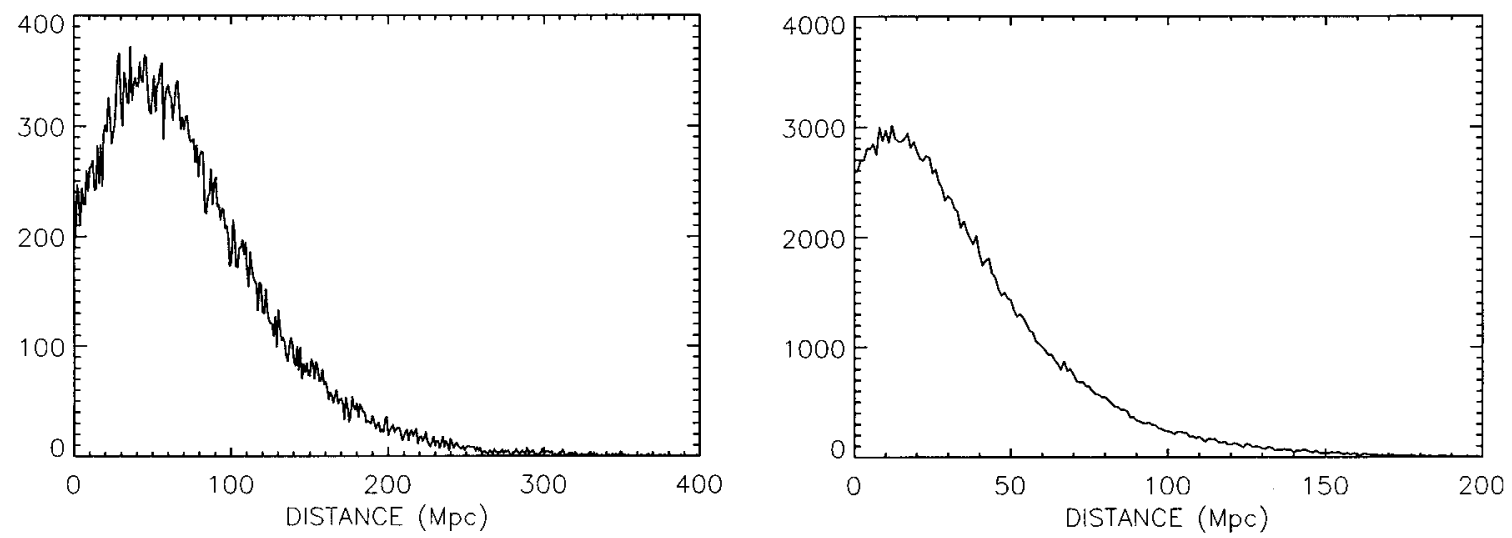

Figure 3-Source distance distributions for the energy bins $0.9-1 \cdot 0 \times 10^{20} \mathrm{eV}$ (left) and $1 \cdot 0-2 \cdot 0 \times 10^{20} \mathrm{eV}$ (right). These indicate the distribution of distances from the sources when protons of this energy are observed.
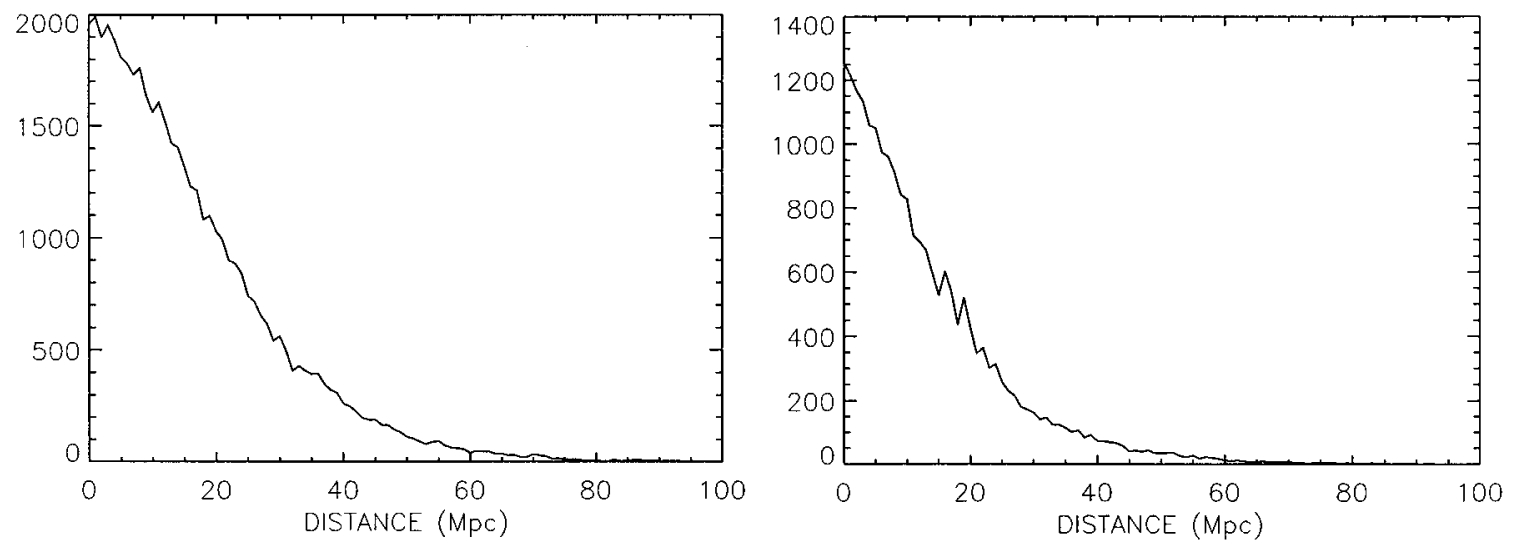

Figure 4-Source distance distributions for the energy bins $2 \cdot 0-3 \cdot 0 \times 10^{20} \mathrm{eV}$ (left) and $3 \cdot 0-4 \cdot 0 \times 10^{20} \mathrm{eV}$ (right).

The calculation was performed for $10^{5}$ protons. The results are given in Table 1 . In this calculation, the energy range from $8 \times 10^{19}$ to $4 \times 10^{20} \mathrm{eV}$ is of particular interest. Source distance distributions for the bins covering this energy range are given in Figures 3 and 4 . To see what the effect on the distributions in these energy bins would be if the source spectrum truncated at a lower energy, the calculation was repeated for a sample of $10^{5}$ protons, with the source spectrum cutting off at $10^{21} \mathrm{eV}$. The result of this calculation is given in Table 2 . It shows that, at energies between $8 \times 10^{19}$ and $4 \times 10^{20} \mathrm{eV}$, there is only a small difference in the statistics when the spectrum truncates at $10^{21} \mathrm{eV}$ instead of $10^{22} \mathrm{eV}$. Not surprisingly, the results are significantly different at higher energies. 
Table 2. Distances from source at various energies for $10^{5}$ particles, with initial energies sampled from an $E^{-2}$ differential spectrum that truncates at $10^{21} \mathrm{eV}$

The $15 \%$ and $85 \%$ points, and the mean, are given to the nearest $\mathrm{Mpc}$

\begin{tabular}{|c|c|c|c|c|}
\hline $\operatorname{Bin}(\mathrm{eV})$ & Number of data values & Mean $(\mathrm{Mpc})$ & $15 \%$ point $(\mathrm{Mpc})$ & $85 \%$ point $(\mathrm{Mpc})$ \\
\hline $8-9 \times 10^{19}$ & 12149 & 101 & 31 & 174 \\
\hline $9-10 \times 10^{19}$ & 9502 & 72 & 21 & 124 \\
\hline $1-2 \times 10^{20}$ & 37873 & 35 & 8 & 62 \\
\hline $2-3 \times 10^{20}$ & 10269 & 14 & 3 & 25 \\
\hline $3-4 \times 10^{20}$ & 4435 & 10 & 2 & 18 \\
\hline $4-5 \times 10^{20}$ & 2254 & 9 & 1 & 16 \\
\hline $5-6 \times 10^{20}$ & 1302 & 7 & 1 & 13 \\
\hline $6-7 \times 10^{20}$ & 841 & 7 & 1 & 12 \\
\hline $7-8 \times 10^{20}$ & 542 & 6 & 1 & 11 \\
\hline $8-9 \times 10^{20}$ & 348 & 5 & 1 & 10 \\
\hline $9-10 \times 10^{20}$ & 204 & 5 & 1 & 9 \\
\hline
\end{tabular}

The results indicate that cosmic rays with energies above $10^{20} \mathrm{eV}$ have a likely origin within about 60 $\mathrm{Mpc}$ of the Earth. Of particular interest here is the likely source distance for cosmic rays of energy $\sim 3 \times 10^{20} \mathrm{eV}$, since this is the highest energy cosmic ray so far observed. Table 1 indicates that there is an $85 \%$ probability that the source for these particles is within $30 \mathrm{Mpc}$ of the Earth.

\section{Discussion}

We have assumed that the spectrum of the highest energy cosmic rays from all sources is a power law with an exponent of $-2 \cdot 0$. Our results will not depend critically on the value of this exponent and we have seen that the effect of truncating the spectrum is not critical at energies presently recorded. Any spectral features which are observed to deviate from a power law will then be propagation effects under this assumption. However, there are other source models which we do not address here which may have quite different spectra. An example of such a model might be topological defects which could produce particles with much greater initial energies (Bhattacharjee, Hill \& Schramm 1992).

At energies below about $10^{20} \mathrm{eV}$, an estimate of the mean propagation distance gives us little useful information in limiting the possible family of cosmic ray sources since it takes us beyond our local supergalactic environment, past $100 \mathrm{Mpc}$ (see Figure 1). However, at energies above $10^{20} \mathrm{eV}$, this is not so. There is strong astronomical evidence that all likely sources within $100 \mathrm{Mpc}$ are closely concentrated in the supergalactic plane (Wall \& Peacock 1985; Shaver 1991) suggesting that this is where we should concentrate our source search. This plane is a remarkable feature with the property that the energetic astronomical objects within $100 \mathrm{Mpc}$ are closely concentrated in it and the concentration is greatest for the most energetic objects (Shaver 1991). It would seem natural to assume that the most energetic objects are the most likely candidates for sources of the highest energy cosmic rays. Objects with large sizes and/or large magnetic fields are probably needed to produce the highest energy cosmic rays and these properties appear to be characteristic of the most energetic radio and X-ray objects. These objects are completely catalogued within $100 \mathrm{Mpc}$.

In looking for sources, we can search through catalogues, such as that of Wall \& Peacock (1985), working down from the most powerful extragalactic objects. However, there is a constraint that we should not move into the regime of conventional galaxies since there is no evidence that our own Milky Way is a source of cosmic rays with energies above $10^{19} \mathrm{eV}$ (Lee \& Clay 1995). We would presumably be swamped by such particles from the plane of the Milky Way if our galaxy, or others like it, were significant sources. It appears that we cannot consider many more objects than those in the catalogues of Shaver or Wall \& Peacock which have lower limits of 1 to $2 \mathrm{Jy}$. This severely limits the possible objects for candidate sources. For instance, assuming a Hubble constant of $50 \mathrm{~km} / \mathrm{s} / \mathrm{Mpc}$, only three of the objects in Wall \& Peacock's catalogue are within $14 \mathrm{Mpc}$, the mean source distance we determined for $3 \times 10^{20} \mathrm{eV}$ protons.

Our present calculation has ignored the effect of the intergalactic magnetic field. The magnitude of this field is unknown as is its structure. It is likely that the field is dominated by a random component, like our own galactic field, and the largest scale of the random component is probably in the region of $100 \mathrm{kpc}$ to $1 \mathrm{Mpc}$ (Ptushkin 1991). This would imply strong scattering for particles up to $10^{20} \mathrm{eV}$ in a one microgauss field and rather rectilinear motion over intergalactic distances for $10^{20} \mathrm{eV}$ particles if the field is below $0.01 \mu \mathrm{G}$. The directional distribution of the highest energy particles should thus give information both on the sources themselves and on the intervening magnetic field.

\section{Conclusions}

Cosmic ray protons of the highest energy propagate to us through the intergalactic medium which is permeated by photons of the $2 \cdot 7 \mathrm{~K}$ microwave back- 
ground. This radiation will act as a target for the cosmic ray particles through photo-pion production and will limit the distances through which those particles can propagate and retain a particular fraction of their original energy. The protons will lose energy at each interaction such that an assumption of the form of the source energy spectrum provides us with a tool to investigate the mean distance a cosmic ray particle with a particular energy will have travelled. We have derived such distances on the basis of a uniform distribution of source locations and a power law source energy spectrum.

The propagation distances are rather small on a cosmological scale. Given the above assumptions, we limit the mean source distance of particles observed with energies over $10^{20} \mathrm{eV}$ conservatively to within $100 \mathrm{Mpc}$, a region dominated by the supergalactic plane. We expect therefore that the sources of the highest energy cosmic rays will be within the supergalactic plane and conclude that a measure of their directional distribution on the sky will allow us both to pin down their likely sources and to put limits on the characteristics of the intergalactic magnetic field.
Aharonian, F. A., \& Cronin, J. W. 1994, Phys. Rev., D 50, 1892

Bhattacharjee, P., Hill, C. T., \& Schramm, D. N. 1992, Phys. Rev. Lett., 69, 567

Bird, D. J., et al. 1993, Phys. Rev. Lett., 71, 3401

Bird, D. J., et al. 1995, ApJ, 441, 144

Elbert, J. W., \& Sommers, P. 1995, ApJ, 441, 151

Geddes, J., Quinn, T. C., \& Wald, R. M. 1996, ApJ, 459, 384

Hill, C. T., \& Schramm, D. N. 1985, Phys. Rev., D 31, 565

Humble, J. E. 1990, Nature, 347, 719

Kewley, L. J., Clay, R. W., \& Dawson, B. R. 1996, Astroparticle Phys., 5, 69

Lee, A. A., \& Clay, R. W. 1995, J. Phys., G 21, 1743

Mather, J. C., et al. 1990, ApJ, 354, L37

Protheroe, R. J., \& Johnson, P. A. 1996, Astroparticle Phys., 4, 253

Ptuskin, V. S. 1991, in Astrophysical Aspects of the Most Energetic Cosmic Rays, ed. M. Nagano \& F. Takahara (Singapore: World Scientific), 112

Shaver, P. 1991, Aust. J. Phys., 44, 759

Stanev, T., et al. 1995, Phys. Rev. Lett., 75, 3056

Szabo, A. P. 1992, PhD Thesis, University of Adelaide

Wall, J. V., \& Peacock, J. A. 1985, MNRAS, 216, 173 